\title{
Prevalence and severity of fatigue in psoriasis and psoriatic arthritis
}

\author{
Magdalena Krajewska-Włodarczyk ${ }^{1}$, Agnieszka Owczarczyk-Saczonek², Waldemar Placek²
}

${ }^{1}$ Department of Internal Medicine, School of Medicine, Collegium Medicum, University of Warmia and Mazury, Olsztyn, Poland 2Department of Dermatology, Sexually Transmitted Diseases and Clinical Immunology, School of Medicine, Collegium Medicum, University of Warmia and Mazury, Olsztyn, Poland

Adv Dermatol Allergol 2020; XXXVII (1): 46-51

DOI: https://doi.org/10.5114/ada.2019.83629

\begin{abstract}
Introduction: Fatigue is an important and underrated symptom of many chronic diseases.

Aim: The evaluation of incidence and severity of fatigue as well as the influence of selected factors on fatigue in patients with psoriasis and psoriatic arthritis (PsA).

Material and methods: The study included 60 patients with PsA, 58 patients with psoriasis and 61 persons in the control group aged 35-70 years. Assessment of fatigue was conducted using a fatigue subscale from the FACIT-F questionnaire. Severity of skin lesions and arthritis was determined with PASI and DAS28, respectively, as well as the number of painful and swollen joints, severity of pain and inflammatory markers.

Results: Severe fatigue occurred in $17 \%, 28 \%$, and $1.6 \%$ of patients with psoriasis, PsA and the control group, respectively. Severity of fatigue was significantly higher in patients with PsA as compared to patients with psoriasis $(p<0.0001)$. In patients with psoriasis and PSA, it decreased along with the duration of psoriasis $(r=0.291, p<0.05$ vs. $r=0.382, p<0.05$, respectively). No significant correlation was found between the duration of PsA and fatigue. After using the linear regression model, severity of fatigue in psoriasis was correlated with the age of patients and the duration of psoriasis, while in PSA, with the duration of psoriasis, PASI, DAS28, CRP and the number of painful joints.

Conclusions: The results of this study may indicate the need for routine fatigue examination among people with psoriasis and psoriatic arthritis.
\end{abstract}

Key words: fatigue, inflammation, psoriasis, psoriatic arthritis.

\section{Introduction}

Fatigue, which is expressed as weakness and lack of motivation, is often a concurrent symptom in chronic inflammatory diseases of joints and skin, systemic connective tissue diseases and other autoimmune diseases [1]. Due to its subjective nature, it is difficult to evaluate, often not understood by patients and their families and overlooked by doctors [2]. The prevalence and severity of fatigue in psoriasis and psoriatic arthritis can be associated with the activity of an inflammatory condition, chronic pain, reduced physical fitness, sleep disturbances, reduced quality of life due to the disease, emotional disorders such as anxiety, depressive reactions and side effects of applied therapy [3]. However, the mechanisms on which the presence of fatigue in psoriasis and pso- riatic arthritis depends have not been well understood. Also, no methods of objective measurement have been developed to determine the severity of fatigue, and all available measuring instruments are based on selfassessment. Due to the simplicity, the most commonly used evaluation scale of fatigue is $100-\mathrm{mm}$ horizontal visual analogue scale (VAS) [4]. Others have also been used, such as the Medical Outcomes Study Short Form 36-item scale (SF-36) [5], the Multidimensional Assessment of Fatigue (MAF) [6], the Functional Assessment of Chronic Illness Therapy - Fatigue (FACIT-F) [7] and the Fatigue Severity Scale (FSS) [8]. All were designed for evaluation of fatigue in chronic diseases. The FACIT-F questionnaire was additionally validated to estimate the fatigue associated with psoriatic arthritis [9]. While using the FACIT-F questionnaire it is necessary to remember

Address for correspondence: Magdalena Krajewska-Włodarczyk PhD, Department of Internal Medicine, School of Medicine, Collegium Medicum, University of Warmia and Mazury, 30 Wojska Polskiego St, 10-900 Olsztyn, Poland, e-mail: magdalenakw@op.pl Received: 25.02.2018, accepted: 8.08.2018. 
that higher values obtained during the test correspond to lower feelings of fatigue.

\section{Aim}

The aim of this work was to compare the incidence of fatigue in patients with psoriasis and psoriatic arthritis, and to assess the influence of selected factors on the severity of fatigue.

\section{Material and methods}

All consecutive patients with psoriasis and with psoriatic arthritis that attended the Rheumatology and Dermatology Clinic and the Department of Rheumatology of the Municipal Hospital in Olsztyn and the Department of Dermatology, Sexually Transmitted Diseases and Clinical Immunology of the University of Warmia and Mazury in Olsztyn, Poland, during a 3-month period (May-July 2017) were enrolled in this study. The study included 60 patients ( 32 men and 28 women) with psoriatic arthritis and 58 patients ( 30 men and 28 women) with psoriasis without arthritis. The control group consisted of 61 people (30 men and 31 women) without psoriasis or psoriatic arthritis. The diagnosis of psoriatic arthritis was established on the basis of CASPAR criteria [10]. The age of the examined subjects varied from 35 to 70 years of age.

Patients with hyperthyroidism and hypothyroidism, as well as with heart, liver or renal insufficiency and malignancies were excluded from the examination. Steroidtreated patients in the last year were also excluded from the study.

In every patient, the following anthropometric parameters were evaluated: body mass index (BMI), waist circumference, and blood pressure. In the dermatological examination, the degree of psoriasis severity was determined using Psoriasis Area Severity Index (PASI) [11]. The activity of joint disease was assessed using the disease activity score of 28 joints (DAS 28) [12] and the number of painful and swollen joints. The VAS scale was used to assess the severity of pain.

The evaluation of fatigue was conducted using the fatigue subscale from the validated Polish version of the FACIT-F questionnaire [13]. In order to use the validated Polish version of the FACIT-F questionnaire in this study, written consent from the FACIT organization was obtained (issued on 30/01/2017). A result of 24 points or less was adopted in order to evaluate the significance of fatigue.

Inflammatory markers were measured with two standard laboratory parameters: erythrocyte sedimentation rate assessed using BD Vacutainer Sedi-15 equipment (BD, Franklin Lakes, NJ, USA) and concentration of C-reactive protein measured with a standard immunoturbidimetric method using a COBAS 6000 INTEGRA apparatus (Roche Diagnostics, Mannheim, Germany).
All patients gave written consent to participation in the examination. The study was conducted in accordance with the recommendations of the Declaration of Helsinki and was approved by the Bioethics Committee of the Warmia and Mazury Chamber of Physicians (OIL 625/16/Bioet; 21.12.2016). A written informed consent was obtained from all patients when they were enrolled.

\section{Statistical analysis}

StatSoft program, Inc. Statistica, version 12.5 (StatSoft, Tulsa, OK, USA) was used for calculations. Obtained results were presented as an arithmetic mean and standard deviation. The Mann-Whitney U-test and the Kruskal-Wallis test were used for comparative analysis between the groups. The presence of the relationship between quantitative features was tested using Pearson's correlation coefficient for parameters consistent with normal distribution and Spearman's correlation coefficient in the case of non-compliance with normal distribution. To evaluate the relationship between the studied data, linear regression modelling was used. Variable models were selected stepwise using backward elimination. The statistical level of significance was $p<0.05$.

\section{Results}

The study included a total of 179 persons aged $35-70$ years, including 60 patients with psoriatic arthritis, 58 patients with psoriasis without arthritis and 61 persons without any inflammatory skin or joint disease. The examined groups did not differ significantly in terms of age or education. Patients with psoriasis without arthritis had a greater severity of skin lesions compared to patients with psoriatic arthritis, but significantly higher values of inflammatory markers were reported in patients with arthritis. The duration of psoriasis in both examined groups did not differ significantly (Table 1). There was a slight unexpected reduction in the severity of fatigue measured in patients with psoriasis and psoriatic arthritis using the FACIT-F fatigue subscale questionnaire, along with the duration of psoriasis ( $r=0.291, p<0.05$ vs. $r=0.382, p<0.05$, respectively). Among patients with psoriatic arthritis no significant correlation was found between the duration of psoriatic arthritis and fatigue. The severity of skin lesions in patients without arthritis did not affect the feeling of fatigue, however such a relation was observed in the group with psoriatic arthritis $(r=-0.58, p<0.05)$. Among all patients, fatigue increased along with pain caused by skin lesions or arthritis ( $r=-0.381, p<0.05$ vs. $r=-0.273, p<0.05$, respectively). In the group with arthritis, the severity of fatigue correlated with the number of painful joints $(r=-0.289, p<0.05)$ as well as with CRP concentration $(r=-0.26, p<0.05)$ and increased ESR $(r=-0.53, p<0.05)$, but not with the number of swollen joints, neither with the occurrence of dactylitis nor the enthesitis type. There was no relationship between the results of the FACIT-F fatigue subscale and the education of 
Table 1. Age and clinical characteristics of the patients studied

\begin{tabular}{|c|c|c|c|c|}
\hline Parameter & $\operatorname{Ps}(n=58)$ & $\operatorname{PsA}(n=60)$ & Control $(n=61)$ & $P$-value \\
\hline Age [years] & $50.7 \pm 10.6$ & $49.7 \pm 8.8$ & $48.4 \pm 10.7$ & NS \\
\hline $\operatorname{Sex}(M / F)$ & $32 / 28$ & $30 / 28$ & $30 / 31$ & NS \\
\hline Ps duration [years] & $17.5 \pm 11.3$ & $16.3 \pm 7.6$ & - & NS \\
\hline PsA duration [years] & - & $7.6 \pm 7.4$ & - & - \\
\hline DAS 28 & - & $3.6 \pm 0.4$ & - & - \\
\hline PASI & $11.3 \pm 6.9$ & $9.7 \pm 8.0$ & - & 0.031 \\
\hline HAQ & $0.76 \pm 0.5$ & $0.85 \pm 0.3$ & - & NS \\
\hline VAS & $24.0 \pm 17.6$ & $33.3 \pm 12.8$ & - & NS \\
\hline TJC & - & $2.9 \pm 1.3$ & - & - \\
\hline SJC & - & $1.3 \pm 0.6$ & - & - \\
\hline Waist circumference $[\mathrm{cm}]$ & $88.1 \pm 13.2$ & $94.2 \pm 15.4$ & $87.1 \pm 15.7$ & NS \\
\hline $\mathrm{BMI}\left[\mathrm{kg} / \mathrm{m}^{2}\right]$ & $27.2 \pm 4.1$ & $28.1 \pm 2.7$ & $26.7 \pm 3.6$ & NS \\
\hline FACIT-F & $38.7 \pm 8.9^{1}$ & $28.7 \pm 8.5$ & $42.0 \pm 7.7$ & $<0.001$ \\
\hline Mtx dose [mg/week] & $13.5 \pm 3.3$ & $17.7 \pm 4.3$ & & 0.039 \\
\hline Mtx duration of treatment [years] & $9.4 \pm 4.1$ & $5.1 \pm 3.2$ & & 0.022 \\
\hline ESR & $13.5 \pm 6.8$ & $25.7 \pm 10.2$ & - & 0.017 \\
\hline CRP $[\mathrm{mg} / \mathrm{dll}]$ & $2.4 \pm 2.5$ & $9.4 \pm 4.1$ & - & $<0.001$ \\
\hline $\mathrm{TSH}[\mathrm{mU} / \mathrm{l}]$ & $2.2 \pm 0.8$ & $2.1 \pm 0.7$ & $2.0 \pm 0.8$ & NS \\
\hline RR systolic [mm Hg] & $129.1 \pm 8.7$ & $134.9 \pm 13.8$ & $129.6 \pm 10.4$ & NS \\
\hline RR diastolic [mm Hg] & $87.5 \pm 7.9$ & $93.2 \pm 10.1$ & $85.3 \pm 8.9$ & NS \\
\hline
\end{tabular}

Results are presented as mean values and standard deviations \pm SD. PS - psoriasis, PSA - psoriatic arthritis, DAS - disease activity score, PASI - psoriasis area severity index, ESR - erythrocyte sedimentation rate, HAQ - health assessment questionnaire, VAS - visual analogue Scale, TJC - tender joint count, SJC - swollen joint count, BMI - body mass index, FACIT-F - Functional Assessment of Chronic Illness Therapy - Fatigue, Mtx - methotrexate, CRP - C-reactive protein, $T S H$ - thyroid-stimulating hormone, $R R$ - blood pressure. ${ }^{I} p=0.04$ psoriasis vs. control.

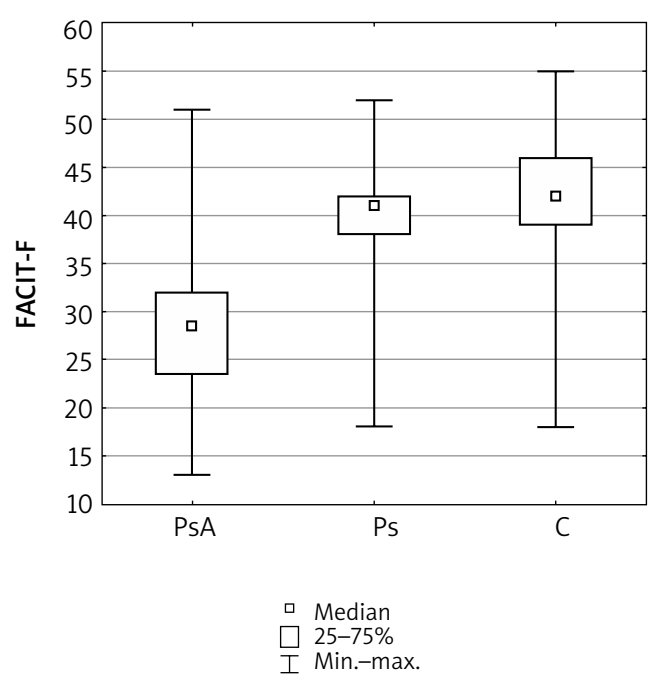

Figure 1. The evaluation of fatigue using FACIT-F questionnaire in patients with psoriatic arthritis, psoriasis and in the control group examined persons. Among patients with psoriatic arthritis, more severe fatigue was observed in women than in men $(p=0.002)$, while in other groups there was no relation between gender and fatigue.

There was no influence of concurrently taken methotrexate on the value of the FACIT-F fatigue subscale in a group of men and women with psoriasis; however, such a relation was observed in women treated for psoriatic arthritis $(r=-0.318, p<0.05)$. Definite increased results of the FACIT-F questionnaire, meaning less fatigue, were obtained in patients treated with anti-tumour necrosis factor- $\alpha$ (anti-TNF- $\alpha)$ antibodies $(p=0.016)$.

More severe fatigue was observed among patients when compared to the control group (Table 1, Figure 1). Severe fatigue, determined in our study to be 24 points or less in the FACIT-F fatigue subscale, was reported in 10 out of 58 patients with psoriasis (17\%) and in 17 out of 60 patients with psoriatic arthritis (28\%). In the control group, severe fatigue was reported in only 1 out of 61 persons (1.6\%).

After using a linear regression model, based on the original model of all potential variables with a matched 
Table 2. The regression coefficients in modelling for fatigue in psoriasis

\begin{tabular}{lcccc}
\hline & Age & Ps duration & HAQ & Adjusted \\
\hline Ps $(n=58)$ & -0.0981 & $0.0210[0.001]$ & -0.5020 & 0.271 \\
& {$[<0.001]$} & & {$[0.017]$} & \\
\hline
\end{tabular}

${ }^{*}$ The $p$-value is shown in brackets. PS - psoriasis, HAQ - health assessment questionnaire.

Table 3. The regression coefficients in modelling for fatigue in psoriatic arthritis

\begin{tabular}{lcccccc}
\hline & Ps duration & PASI & DAS 28 & CRP & TJC & Adjusted $R^{2}$ \\
\hline PsA & $0.362[<0.001]$ & $-0.340[<0.001]$ & $-0.271[<0.001]$ & -0.184 & $-0.166[0.006]$ & 0.981 \\
& & & & {$[0.002]$} & \\
\hline
\end{tabular}

*The $p$-value is shown in brackets. PSA - psoriatic arthritis, PS - psoriasis, PASI - psoriasis area severity index, DAS - disease activity SCOre, CRP - C-reactive protein, TJC-tender joint count, SJC-swollen joint count.

stepwise backward elimination, factors were determined that had the greatest impact on the total severity of fatigue in examined patients. There was a significant correlation between fatigue in people with psoriasis, and the age of patients and the duration of skin disease. The severity of fatigue corresponded with a reduced quality of life as assessed by the HAQ questionnaire (Table 2). In the group with psoriatic arthritis, fatigue was significantly correlated with the duration of psoriasis, the severity of skin lesions assessed by PASI, the activity of arthritis assessed by DAS 28, the CRP concentration, and the number of painful joints (Table 3).

\section{Discussion}

Chronic fatigue associated with psoriasis and psoriatic arthritis may constitute a serious, although underrated problem in clinical practice $[14,15]$. So far, little research has been carried out on fatigue in psoriasis. In our study we have discovered severe fatigue in $17 \%$ of patients with psoriasis, and it was correlated with their age, the duration of psoriasis and a reduced quality of life. In the study conducted by Verhoeven et al., severe fatigue was reported in $25 \%$ of patients with psoriasis [15]. In a recently published study conducted by Skoie et al., the severity of fatigue in psoriasis was associated with depression, pain and smoking addiction, but, contrary to expectations, it did not depend on the severity of psoriasis; which was also noted in our study [14]. The lack of correlation between the disease activity assessed using PASI was also described by Tobin et al., but twice as often severe fatigue was assessed in patients treated systemically than in patients treated with phototherapy [16]. Different results were presented by Verhoeven et al., who reported a strong correlation between assessed fatigue and reduced quality of life associated with disease, as well as with an assessment of the severity by patients [15]. The incidence of fatigue in patients with psoriasis can be associated with anxiety and other symptoms of depression [16, 17], and according to some researchers, it is more common in women than men [16]. In the group of examined patients with psoriasis, we have not noted a correlation between gender and severity of fatigue. It seems that skin changes in psoriasis, especially active psoriasis, causes local pain and itching, which leads to a secondary sleep disorder. This clearly must also affect the severity of fatigue [18], however in our study we did not observe a correlation between the severity of skin lesions assessed with PASI and fatigue. In our study, the use of methotrexate was not associated with severity of fatigue in patients with psoriasis, but significantly lower fatigue was observed among people treated with anti-TNF- $\alpha$ antibodies. There is only little available data in the literature concerning the correlation of psoriasis therapy with decreased feelings of fatigue. Most of the data concerning treatment effects of fatigue reduction in psoriasis were obtained during clinical trials with biological drugs, mainly with molecules such as anti-TNF- $\alpha$. According to patients, infliximab, adalimumab, etanercept and ustekinumab significantly reduce fatigue, whereas discontinuation was associated with severe symptoms [18-28].

We have observed severe fatigue in $28 \%$ of patients with psoriatic arthritis, similar to studies conducted by Husted et al. and Tobin et al., where severe fatigue was observed in about $30 \%$ of patients with psoriatic arthritis $[3,16]$. In our study, fatigue in psoriatic arthritis has not been associated with duration of the joint disease, and was much more severe than among patients with psoriasis, which may indicate that fatigue may be one of the potential forecasting factors to predict development of psoriatic arthritis. In a recently published study on nonspecific symptoms prior to incidence of arthritis associated with psoriasis, the severity of fatigue in patients with psoriasis was associated with a higher risk of psoriatic arthritis [29]. In our study, we have discovered a significant correlation between pain intensity during arthritis and fatigue, similar to a study by Husted et al. [30]. Among our examined patients with psoriatic arthritis, the fatigue estimated by the FACIT-F fatigue subscale was significantly correlated with values of the severity of PASI and DAS28, as well as with the number of painful, but not swollen joints. Similar results were presented by Gudu et al., who described a significant correlation be- 
tween the presence and severity of fatigue with psoriasis severity and number of painful joints. In addition, different than in our study, the authors reported a relation of the severity of fatigue with lower levels of education and the presence of enthesopathy [31]. In one of the studies, a greater feeling of fatigue was reported by women with psoriasis [16]. Among women with psoriatic arthritis examined by us, the fatigue was also significantly higher ( $p=0.024)$ than in the group of men, while among men a greater activity of disease was observed, which was assessed using DAS28 ( $p=0.311)$, higher CRP concentrations $(p=0.427)$ and a larger number of painful joints $(p=0.042)$.

It is also necessary to remember that fatigue can be associated with side effects of traditional disease modifying drugs, such as methotrexate and leflunomide [32]. Among women with psoriatic arthritis in our study, the fatigue significantly increased during methotrexate therapy $(p=0.037)$, we did not observe such a correlation among women with psoriasis, which could be caused by lower methotrexate doses applied in the treatment of skin disease $(p=0.045)$.

In our study, both among patients with psoriasis and psoriatic arthritis, the fatigue decreased unexpectedly along with the duration of psoriasis, which may be a result of effectively conducted treatment or the adaptation of patients to symptoms. However, among patients with psoriatic arthritis, the duration did not affect the feeling of fatigue. Taking into account the fact that among examined patients with psoriatic arthritis, the fatigue was significantly higher than among people with psoriasis, this may suggest that fatigue during psoriasis is not only one of the symptoms, but can also be a predictive factor for the development of psoriatic arthritis.

The problem of fatigue for patients seems to be very important, which additionally results from the study conducted by Gudu et al., in which patients with psoriatic arthritis, irrespective of the place of residence and cultural factors, have reported fatigue as the most common symptom, after pain, even more annoying than the severity of psoriatic skin lesions [31].

\section{Conclusions}

The treatment of psoriasis and psoriatic arthritis aims to reduce the inflammatory process, pain relief and to prevent organ damage. However, this traditional therapeutic approach does not cover the treatment of fatigue. Although the quality of life in patients with psoriasis and psoriatic arthritis has already been the subject of many studies, little attention has been paid to the presence and severity of fatigue, which in many cases is a part of the clinical image in psoriasis and psoriatic arthritis. Yet, fatigue which is an expression of weakness and lack of motivation may lead to loss of employment, social isolation and reduced quality of life as a condition of this disease. A few methods of fatigue self-assessment by patients based on a visual analogue scale or detailed questionnaires have been used in psoriasis and psoriatic arthritis, but more often they have been used in scientific research than during daily clinical practice. In our study, severe fatigue significantly affecting the lives of patients was observed in $17 \%$ of persons with psoriasis and in $28 \%$ of persons with psoriatic arthritis, which justifies routine fatigue examination among people with psoriasis and psoriatic arthritis. However, factors and mechanisms which link severity of fatigue in psoriasis with the development of psoriatic arthritis require further multidirectional research.

\section{Conflict of interest}

The authors declare no conflict of interest.

\section{References}

1. Norheim KB, Jonsson G, Omdal R. Biological mechanisms of chronic fatigue. Rheumatology (Oxford) 2011; 50: 1009-18.

2. Poulson MJ. Not just tired. J Clin Oncol 2001; 19: 4180-1.

3. Husted JA, Tom BD, Schentag CT, et al. Occurrence and correlates of fatigue in psoriatic arthritis. Ann Rheum Dis 2009; 68: 1553-8.

4. Wolfe F, Michaud K, Pincus T. Preliminary evaluation of a visual analog function scale for use in rheumatoid arthritis. J Rheumatol 2005; 32: 1261-6.

5. Ware JE, Kosinski M, Keller SD. SF-36 Health Survey: Manual and Interpretation Guide. MA, USA: The Health Institute, New England Medical Center; 1983.

6. Belza BL, Henke CJ, Yelin EH, et al. Correlates of fatigue in older adults with rheumatoid arthritis. Nurs Res 1993; 42: 93-9.

7. Cella D, Yount S, Sorrensen M, et al. Validation of the Functional Assessment of Chronic Illness Therapy Fatigue Scale relative to other instrumentation in patients with rheumatoid arthritis. J Rheumatol 2005; 32: 811-19.

8. Krupp LB, LaRocca NG, Muir-Nash J, Steinberg AD. The fatigue severity scale. Application to patients with multiple sclerosis and systemic lupus erythematosus. Arch Neurol 1989; 46: 1121-3.

9. Chandran V, Bhella S, Schentag C, et al. Functional assessment of chronic illness therapy-fatigue scale is valid in patients with psoriatic arthritis. Ann Rheumatic Dis 2007; 66: 936-9.

10. Taylor W, Gladman D, Helliwell P, et al. Classification criteria for psoriatic arthritis: development of new criteria from a large international study. Arthritis Rheum 2006; 54: 266573.

11. Mrowietz U, Kragballe K, Reich K, et al. Definition of treatment goals for moderate to severe psoriasis: a European consensus. Arch Dermatol Res 2011; 301: 1-10.

12. Praevoo ML, van't Hof MA, Kuper HH, et al. Modified disease activity scores that include twenty-eight-joint counts. Arthritis Rheum 1995; 38: 44-8.

13. Www.facit.org/FACITOrg/Questionnaires (access on 30/01/2017).

14. Skoie IM, Dalen I, Ternowitz T, et al. Fatigue in psoriasis a controlled study. Br J Dermatol 2017; 177: 505-12. 
15. Verhoeven EW, Kraaimaat FW, van de Kerkhof PC, et al. Prevalence of physical symptoms of itch, pain and fatigue in patients with skin diseases in general practice. Br J Dermatol 2007; 156: 1346-9.

16. Tobin AM, Sadlier M, Collins P, et al. Fatigue as a symptom in psoriasis and psoriatic arthritis: an observational study. Br J Dermatol 2017; 176: 827-8.

17. McDonough E, Ayearst R, Eder L, et al. Depression and anxiety in psoriatic disease: prevalence and associated factors. J Rheumatol 2014; 41: 887-96.

18. Thaci D, Galimberti R, Amaya-Guerra M, et al. Improvement in aspects of sleep with etanercept and optional adjunctive topical therapy in patients with moderate-to-severe psoriasis: results from the PRISTINE trial. J Eur Acad Dermatol Venereol 2014; 28: 900-6.

19. Krueger GG, Langley RG, Finlay AY, et al. Patient-reported outcomes of psoriasis improvement with etanercept therapy: results of a randomized phase III trial. Br J Dermatol 2005; 153: 1192-9.

20. Reich K, Nestle FO, Papp K, et al. Improvement in quality of life with infliximab induction and maintenance therapy in patients with moderate-to-severe psoriasis: a randomized controlled trial. Br J Dermatol 2006; 154: 1161-8.

21. Tyring S, Gottlieb A, Papp K, et al. Etanercept and clinical outcomes, fatigue, and depression in psoriasis: double-blind placebo-controlled randomised phase III trial. Lancet 2006; 367: 29-35.

22. Krishnan R, Cella D, Leonardi C, et al. Effects of etanercept therapy on fatigue and symptoms of depression in subjects treated for moderate to severe plaque psoriasis for up to 96 weeks. Br J Dermatol 2007; 157: 1275-7.

23. Daudén E, Griffiths CE, Ortonne JP, et al. Improvements in patient-reported outcomes in moderate-to-severe psoriasis patients receiving continuous or paused etanercept treatment over 54 weeks: the CRYSTEL study. J Eur Acad Dermatol Venereol 2009; 23: 1374-82.

24. Reich K, Segaert S, Van de Kerkhof P, et al. Once-weekly administration of etanercept $50 \mathrm{mg}$ improves patient-reported outcomes in patients with moderate-to-severe plaque psoriasis. Dermatology 2009; 219: 239-49.

25. Lebwohl M, Papp K, Han C, et al. Ustekinumab improves health-related quality of life in patients with moderate-tosevere psoriasis: results from the PHOENIX 1 trial. Br J Dermatol 2010; 162: 137-46.

26. Papp K, Crowley J, Ortonne JP, et al. Adalimumab for moderate to severe chronic plaque psoriasis: efficacy and safety of retreatment and disease recurrence following withdrawal from therapy. Br J Dermatol 2011; 164: 434-41.

27. Nakagawa H, Schenkel B, Kato M, et al. Impact of ustekinum$a b$ on health-related quality of life in Japanese patients with moderate-to-severe plaque psoriasis: results from a randomized, double blind, placebo-controlled phase 2/3 trial. J Dermatol 2012; 39: 761-9.

28. Kalb RE, Blauvelt A, Sofen HL, et al. Effect of infliximab on health-related quality of life and disease activity by body region in patients with moderate-to-severe psoriasis and inadequate response to etanercept: results from the PSUNRISE trial. J Drugs Dermatol 2013; 12: 874-80.

29. Eder L, Polachek A, Rosen CF, et al. The development of PsA in patients with psoriasis is preceded by a period of nonspecific musculoskeletal symptoms: a prospective cohort study. Arthritis Rheumatol 2017; 69: 622-9.

30. Husted JA, Tom BD, Farewell VT, et al. Longitudinal analysis of fatigue in psoriatic arthritis. J Rheumatol 2010, 37: 1878-84.
31. Gudu T, Etcheto A, de Wit M, et al. Fatigue in psoriatic arthritis - a cross-sectional study of 246 patients from 13 countries. Joint Bone Spine 2016; 83: 439-43.

32. Singh $\mathrm{H}$, Arya S, Talapatra P, et al. Assessment of fatigue in rheumatoid arthritis (by Functional Assessment of Chronic Illness Therapy-Fatigue score) and its relation to disease activity and anemia. J Clin Rheumatol 2014; 20: 87-90. 